\title{
ADSORPTION OF DIMERIZING FLUIDS ON SOLID SURFACES. MODELS OF OVERLAPPING SPHERES
}

\author{
D. Henderson ${ }^{1}$, O. Pizio ${ }^{2, *}$, S. Sokołowski ${ }^{3}$, A. Trokhymchuk ${ }^{2, *}$ \\ ${ }^{1}$ Department of Chemistry and Biochemistry, Brigham Young University, Provo, Utah 84602, USA \\ ${ }^{2}$ Instituto de Química de la UNAM, Coyoacán, 04510, México D.F., México \\ ${ }^{3}$ Faculty of Chemistry, MCS University, Lublin 20031, Poland \\ * Permanent address: Institute for Condensed Matter Physics, \\ National Academy of Sciences of the Ukraine, 290011 Lviv, Ukraine
}

(Received October 17, 1995)

\begin{abstract}
Recent studies of inhomogeneous associating fluids are reviewed. The presentation is restricted to the case of the adsorption of dimerizing fluids on solid surfaces. Structureless and crystalline surfaces, which are both reactive and nonreactive, are considered. The integral equation methods are our basic tool but some results of Monte Carlo simulations are given as well.

Keywords: surface, association, adsorption, density profiles.
\end{abstract}

PACS number(s): 68.45.-V; 61.20.Qg

\section{INTRODUCTION}

In this paper we intend to review and summarize some recent developments in the theory of inhomogeneous associating fluids. In addition, some original results are presented and some problems which must be solved in future studies are discussed.

Inhomogeneous associating fluids, abbreviated in what follows as IAFs, represent a challenging subject of investigation in statistical mechanics. Several important problems of chemistry and chemical engineering involve IAFs. Adsorption and coadsorption, chemisorption and corrosion, heterogeneous catalysis on crystalline and noncrystalline materials, chemical reactions at gas-liquid and liquid-liquid intefaces are particular examples involving inhomogeneous chemically associating (reacting) fluids. Such IAFs are difficult to study. They combine all the complexity of the description of the bulk associating fluids with the additional difficulties of the interface.

The phenomena which occur in IAFs are much richer than those which take place in the bulk. These phenomena are complex because of nonassociative interactions and of the specific features of the associative interactions and of inhomogeneity. Therefore, it is necessary to introduce simplifications which provide the possibility of obtaining an analytical or a numerical solution. Only the simplest models of IAFs which give insight on a restricted set of interfacial phenomena have been studied. However, a successful desription of adsorption can be reached, even within the framework of such simplified theoretical procedures. In this study we restrict ourselves to the case of an associating fluid in contact with the solid surface. Association in a liquid-liquid interface is not considered.

The theory of IAFs has benefited from the methods and results of the theory of the bulk associating fluids (AFs). It is a rapidly developing area, especially during the last decade. The most important earlier contributions are due to Andersen, Chandler and Pratt, Høye and Olaussen [1-3]. In 1984 Cummings and Stell [4] and Wertheim [5] initiated systematic studies of associating bulk fluids. In Ref. [4], and subsequent series of papers of Stell and collaborators [6-8], a model of hard spheres with a spherically symmetric associative potential has been examined by means of the standard Ornstein-Zernike (OZ) equation with standard liquid state closures. This approach was applied immediately to ionic associating fluids by Rasaiah et al. [9-12]; later more sophisticated models with electrostatic interactions have been studied $[13,14]$.

However, subsequent developments showed that the usual liquid state closures lead to deviations from the law of mass action and are not completely adequate for the calculation of the degree of association on the strength of associative interaction and the density of the fluid. To overcome this difficulty, Stell and Zhou [1517] have proposed a thermodynamic scheme to evaluate the equilibrium association constant. If one focuses on the determination of the structural properties, this thermodynamic scheme must be introduced into the theory. Particular examples of this approach are the extended mean spherical approximation (EMSA) and the "sitesite" EMSA which uses the "site-site" OZ equation reformulated for the particle-particle correlations and for the incomplete association [18-20]. These approximations have been used for the heterogeneous dimerization reaction $(A+B \rightleftharpoons A B)$.

The theory of Wertheim [5, 21-23] is constructed in a different way. It uses a fugacity expansion as a cornerstone, in contrast to the method briefly reviewed above. Diagrammatic expansions of the grand partition sum and one-particle distribution function then permit the distinction of the densities of the nonbonded and bonded particles. The $\mathrm{OZ}$ equation is reformulated in terms of these densities and an OZ-like equation results. The particle-particle correlation functions are given as linear combinations of the partial correlation functions which correspond to those nonbonded and bonded species. This theory has been exploited extensively by different groups 
to study nonionic and ionic associating systems in a set of recent publications [24-29]. Originally the theory of Wertheim was developed for a highly directional associative interactions. Later, Stell and Kalyuzhnyi have extended this approach for a spherically symmetric interactions $[20,30]$. We will not make a detailed overview of the theory of the bulk systems as our aim is to discuss IAFs.

The study of IAF's has been initiated very recently. Different methods have been applied. Kierlik and Rosinberg [31-33] have constructed a density functional approach for IAFs on the basis of Wertheim's theory. However, they considered only systems in which the monomers cannot penetrate each other, i.e. there is no contraction of the volume during the formation of multimers. Similar dimerizing models have been considered in Refs. $[34,35]$ by means of the associative analogue of the Henderson-Abraham-Barker (HAB) integral equation [36, 37]. We have investigated those models which permit a contraction of the volume of dimers in respect to the monomers [38-46]. These models are reviewed in the present paper.

Two levels of the description of inhomogeneous simple fluids [37] have been developed, namely the singlet level theory (SLT) and the pair level theory (PLT). The SLT is simpler than the PLT and, as a result, has been most frequently applied to IAFs. To our best knowledge, the only attept to implement the PLT for IAFs has been undertaken in Ref. [46]. Therefore, in what follows we emphasize the SLT and the results obtained from this method. Structureless and crystalline surfaces are discussed in detail. The PLT is discussed more briefly. In addition, we present some Monte Carlo simulation results.

\section{SINGLET LEVEL THEORY FOR DIMERIZING FLUIDS ON THE NONCRYSTALLINE SURFACES}

\section{A. General consideration}

The singlet level theory is the most straightforward way to obtain the density profiles (DPs). We shall use it in this and following sections. The models under study are described by the following potential energy

$$
U_{p o t}=\sum_{m, n=A, B} \sum_{i<j} U_{m n}\left(r_{i j}\right)+\sum_{m=A, B} \sum_{i} U_{m}\left(z_{i}\right)
$$

where the first term is the potential energy of the bulk fluid and the second term corresponds to the fluidsurface interactions. It is chosen as

$$
U_{m}(z)=\left\{\begin{array}{c}
\infty, \quad z \leq 0 \\
0, \quad z>0
\end{array}\right.
$$

The planar, impenetrable, wall is formed as a result of a limiting procedure in which the concentration of the wall particle species is taken to tend to zero whereas the diameter of the wall particles tends to infinity [36]. This is the so-called wall limit which provides the singlet level description of the interfacial properties. The singlet level Percus-Yevick (PY1) and singlet hypernetted chain equation (HNC1) are the most popular approximations for the DPs. They read, respectively

$$
\begin{aligned}
& y_{m}\left(\mathbf{r}_{1}\right)=1 \\
& +\sum_{n} \rho_{n} \int d \mathbf{r}_{2} c_{m n}\left(\left|\mathbf{r}_{1}-\mathbf{r}_{2}\right|\right)\left[y_{n}\left(\mathbf{r}_{2}\right) \gamma_{n}\left(\mathbf{r}_{2}\right)-1\right] \\
& y_{m}\left(\mathbf{r}_{1}\right) \\
& =\exp \left\{\sum_{n} \rho_{n} \int d \mathbf{r}_{2} c_{m n}\left(\left|\mathbf{r}_{1}-\mathbf{r}_{2}\right|\right)\left[y_{n}\left(\mathbf{r}_{2}\right) \gamma_{n}\left(\mathbf{r}_{2}\right)-1\right]\right\}
\end{aligned}
$$

where $y_{m}(\mathbf{r})$ is the one-particle cavity distribution function, $\gamma_{m}(\mathbf{r})$ is the Boltzmann factor, $\gamma_{m}(\mathbf{r})=$ $\exp \left[-\beta U_{m}(\mathbf{r})\right]$, and $c_{m n}(r)$ are the direct correlation functions of the bulk fluid. The HNC approximation consists in setting the bridge function, $B(\mathbf{r})$, equal to zero. The $c_{m n}(r)$ represent the only input necessary to obtain the DPs, $\rho_{m}(\mathbf{r}), \rho_{m}(\mathbf{r})=\rho_{m} g_{m}(\mathbf{r})=\rho_{m} y_{m}(\mathbf{r}) \gamma_{m}(\mathbf{r})$. Before describing the models for the bulk associating fluids it is worth noting that for the $\delta$-like (sticky) associative interactions, used in the majority of the previous studies, the direct correlation functions contain a term proportional to the $\delta$-function which must be treated analytically while solving the Eqs. (2.3), (2.4) numerically. This $\delta$-type interaction results from the transformation proposed by Baxter [47] which is employed to yield an analytical solution of the model AF. In the case of a square well associative interaction, the singlet level can be solved numerically without difficulty. We prefer to use the square well interaction and a numerical solution of the integral equations for the IAFs, because numerical methods are required when more sophisticated forms for the interparticle and particle-surface interactions are considered.

\section{B. Bulk input into the singlet level theory}

We focus on the model of Cummings and Stell [4] proposed for heterogeneous association, $A+B \rightleftharpoons A B$. It is defined by the following interaction potentials

$$
U_{A A}(r)=U_{B B}(r)=\left\{\begin{array}{c}
\infty, r<\sigma_{f} \\
0, \quad r>\sigma_{f}
\end{array}\right.
$$

and

$$
U_{A B}(r)=U_{B A}(r)
$$




$$
=\left\{\begin{array}{cc}
\infty, & r<L_{b}-0.5 w \\
-\varepsilon_{b}, & L_{b}-0.5 w<r<L_{b}+0.5 w \\
\varepsilon_{m}, & L_{b}+0.5 w<r<\sigma_{f} \\
0, & r>\sigma_{f}
\end{array},\right.
$$

where $\sigma_{A}=\sigma_{B}=\sigma_{f}$ is the diameter of the spherically symmetric monomers (without any loss of generality we assume $\sigma_{f}=1$ ). The associative interaction between the $A$ and $B$ species has the form of a square well with the width $w$ located at the bonding distance $L_{b}$. The strength of the associative interaction is given by the depth of the square well $\varepsilon_{b}$. The height of the square mound $\varepsilon_{m}$ is chosen to satisfy the condition $e^{-\beta \varepsilon_{m}} \approx 0$. The associative interaction is spherically symmetric and for $L_{b}+0.5 w<1 / 2$, steric saturation occurs at the dimer level, i.e. higher order complexes are not formed. This model has been solved analytically under equimolar conditions $\left(\rho_{A}=\rho_{B}=\rho / 2, \rho\right.$ is the total density of parti- cles) by means of the Percus-Yevick (PY) approximation [4], the extended mean spherical approximation (EMSA) [20], a reformulated site-site EMSA, and the associative Percus-Yevick approximation (APY) [5]. An analytical solution can be obtained only by a transformation of the square well potential to a delta function. However, as mentioned above, the importance of obtaining an analytical solution decreases if one needs to apply the bulk direct correlation functions in Eqs. (2.3), (2.4) because even for quite simple fluid-solid potentials, Eqs. (2.3), (2.4) must be solved numerically. A numerical solution of the equations for the bulk model is more convenient when the square well associative interaction is in the form given by Eq. (2.6). However, we have performed calculations of the DPs for the models with intracore sticky potential by using the PY approximation [38-41]. In order to omit problems arising in the evaluation of the degree of association, a more sophisticated theory provided by the EMSA has been applied [43]. The EMSA consists of the $\mathrm{OZ}$ equation

$$
h_{m n}\left(r_{12}\right)-c_{m n}\left(r_{12}\right)=\sum_{k} \rho_{k} \int d \mathbf{r}_{3} h_{m k}\left(r_{13}\right) c_{k n}\left(r_{32}\right)
$$

and closure relations

$$
\begin{gathered}
h_{m n}(r)=-1+\left(1-\delta_{m n}\right) \frac{\Lambda_{b}}{4 \pi L_{b}^{2} \sqrt{\rho_{m} \rho_{n}}} \delta\left(r-L_{b}\right), r<1, \\
c_{m n}(r)=(-1)^{\delta_{m n}} \frac{3\left[\Lambda_{b}\left(1-\delta_{m n}\right)+\delta_{m n}\right]}{4 \pi r L_{b}^{2} \sqrt{\rho_{m} \rho_{n}}} e^{-K r}, r>1,
\end{gathered}
$$

where $K$ is the correlation length dependent on the degree of dimerization $\Lambda_{b}$ in the following way

$$
K^{2}=\frac{3\left(1-\Lambda_{b}^{2}\right)}{\left(\Lambda_{b} L_{b}\right)^{2}}
$$

The EMSA requires the degree of dimerization $\Lambda_{b}$ as an input parameter. We calculate it according to the scheme used in Ref. [20] and modified for a twocomponent case (see [43] for more details). The direct correlation functions obtained from the numerical solution of the EMSA are then used in the PY1 approximation to calculate the DPs.

The reformulated "site-site" EMSA (SSEMSA) approximation is a more refined treatment. Actually, Eqs. (2.8) and (2.9) constitute a simplified version of the SSEMSA. The SSEMSA comprises following closures

$$
h_{m n}(r)=-1+\frac{\omega_{m n}(r)}{\sqrt{\rho_{m} \rho_{n}}}, r<1
$$

and

$$
c_{m n}(r)=-\frac{\left[\omega^{-1}\right]_{m n}}{\sqrt{\rho_{m} \rho_{n}}}, r>1
$$

where $\omega_{m n}(r)=\left(1-\delta_{m n}\right) \Lambda_{b} F^{(a s)}(r) / K_{0 b}$ is the intramolecular structure factor under off-confinement conditions [19], $F^{(a s)}(r)=f_{A B}(r)-f_{A B}^{(h s)}(r)$ is the difference between the Mayer functions of the model with associative interactions and a corresponding hard sphere model [20]. The parameter $K_{0 b}$ is the equilibrium association constant of dimerization reaction at infinite dilution. It can be expressed in terms of the parameters of the square-well associative interaction as follows [4] 


$$
K_{0 b}=\frac{\pi}{3} e^{\beta \varepsilon_{b}}\left[12 L_{b}^{2} w+w^{3}\right]
$$

The elements of the reverse matrix in Eq. (2.12) are calculated numerically for the square-well associative interaction. Actually, the EMSA closure given by Eq. (2.9) includes the long-range asymptotics of the SSEMSA, Eq. (2.12). The EMSA and SSEMSA results coincide for high densities. The EMSA becomes less adequate at lower densities, in contrast to the SSEMSA which agrees well with the simulation data for a wide range of densities [20].

The SSEMSA is the most sophisticated approach developed within the framework of the theory of Stell et al. for the description of structural properties. It can be used successfully as input into the singlet level equations for the density profiles. Some of the results which involve the bulk SSEMSA are presented in the next section.

As we have noted in the description of the interparticle interactions, when $L_{b}+0.5 w>0.5$ the spherically symmetric associative interaction in the models for homogeneous and heterogeneous association [4] allow for the formation of not only dimers but also of chain-like and branched structures made of monomeric units. To generate longer dimers without chains and branched structures, one must use a highly directional associative interaction.

Therefore it is worth discussing the model for association which comprises a highly directional associative interaction and serves to demonstrate an application of the theory of Wertheim. Study homogeneous association for simplicity. Consider in contrast to the intracore nonsticky associative shell investigated above, a onecomponent shielded sticky point model for an associating fluid. This was introduced originally in Refs. [5, 48] and has been studied by us near a hard wall in Ref. [42]. It is characterized by the following interparticle interaction

$$
U(1,2)=U^{(r e f)}\left(\left|\mathbf{r}_{12}\right|\right)+U^{(a s)}\left(x_{12}\right),
$$

where $x_{12}=\left|\mathbf{r}_{12}+\mathbf{d}\left(\Omega_{1}\right)-\mathbf{d}\left(\Omega_{2}\right)\right|, \mathbf{d}(\Omega)$ denotes the position and orientation of the interaction site located inside a hard core of the particles determined by $U^{(r e f)}\left(\left|\mathbf{r}_{12}\right|\right)$

$$
U^{(r e f)}\left(\left|\mathbf{r}_{12}\right|\right)=\left\{\begin{array}{cc}
\infty, & r_{12}<L_{b} \\
\varepsilon_{m}, & L_{b}<r_{12}<\sigma_{f} \\
0, & r_{12}>\sigma_{f}
\end{array}\right.
$$

and where the associative potential is

$$
U^{(a s)}(x)=\left\{\begin{array}{cc}
-\varepsilon_{b}, & x<a \\
0, & x>a
\end{array}\right.
$$

Some of the notation has been introduced before and need not be explained again. The parameter $a$ describes the range of action of the associative potential. In order to ensure steric saturation at the dimer level, one must choose the bonding distance $L_{b}$, the position of the intracore site $d(d=|\mathbf{d}|)$ and range of associative potential $a$ in a specific manner, namely $L_{b}<2 d+a<L_{b}-(2-\sqrt{3}) d$. This model permits an analytical solution in the delta function limit (see, eg. [48] for more detail), which is similar to the transformation of a square well into the $\delta$-type potential according to the recipe of Baxter [47]. An analytic solution has been obtained using Wertheim's theory in the associative Percus-Yevick approximation. This provides partial pair correlation functions $h_{\alpha \beta}(r)$ $(\alpha, \beta=0,1$ for nondimerized and dimerized particles, respectively) which form the familiar total correlation function

$$
\begin{aligned}
& h(r)=h_{00}(r) \\
& +\left(1-\Lambda_{b}\right) h_{01}(r)+\left(1-\Lambda_{b}\right)^{2} h_{11}(r) .
\end{aligned}
$$

As previously, $\Lambda_{b}$ is the degree of dimerization. One can apply either $h(r)$ or $c(r)$ as an input for the singlet level theory of associating fluids.

Usually the direct correlation function is used for numerical solutions because it is shorter-ranged than the pair correlation function. The direct correlation function follows from the numerical solution of the standard OZ equation with the given $h(r)$. This is the scheme applied in Ref. [42] to evaluate the DPs of particles near a hard wall and in a slit-like pores.

With these comments we terminate the description of the bulk models. In the next section the density profiles obtained for models with different forms of the onedimensional external field $U_{m}(z)$ are discussed.

\section{Density profiles for associating fluids near structureless surfaces}

Consider first the case of a hard wall. This is the simplest model which includes the geometrical constraints of the solid surface. We postpone investigation of more sophisticated fluid-solid interactions (Yukawa type, etc.). The hard wall case is determined by $U_{m}(z)$ given by Eq. (2.2), i.e. a nonassociative hard wall is located at $z=0$. The nonassociative adsorption on the idealized structureless surface is studied. The DPs of a heterogeneously dimerizing fluid (the model of Cummings and Stell [4]) are presented in Figs 1 and 2. They have been calculated within the framework of the PY1/EMSA and HNC1/EMSA approximations. We have chosen here $L_{b}=0.42$.

The model is studied at high densities ( $\rho=0.646$ (Fig. 1) and $\rho=0.821$ (Fig. 2)) and high degrees of dimerization $\left(\beta \varepsilon_{b}=4.25\right.$, see Fig. 1 of Ref. [43]). Therefore, possible inaccuracies of the EMSA are minimized. A comparison with our MC simulation data [49] shows that, in general, the PY1 and HNC1 approximations provide an adequate description of the DPs. Some inaccuracies 
are observed in the vicinity of the wall. It is worth noting that for increasing density, the contact adsorption of the AF under study increases, similarly to the case of a hard sphere fluid. The oscillating behaviour of the DPs for high packing fractions and for high degrees of association reflects the tendency for the formation of a multilayer structure near a hard wall. The cusp at $z \approx L_{b}$, demonstrates the presence of dimer species in the adlayer. A comparison with the MC data shows that the HNC1/EMSA approximation overestimates the contact value of the DP whereas the PY1/EMSA underestimates it slightly. In general the PY1 approximation is preferable.

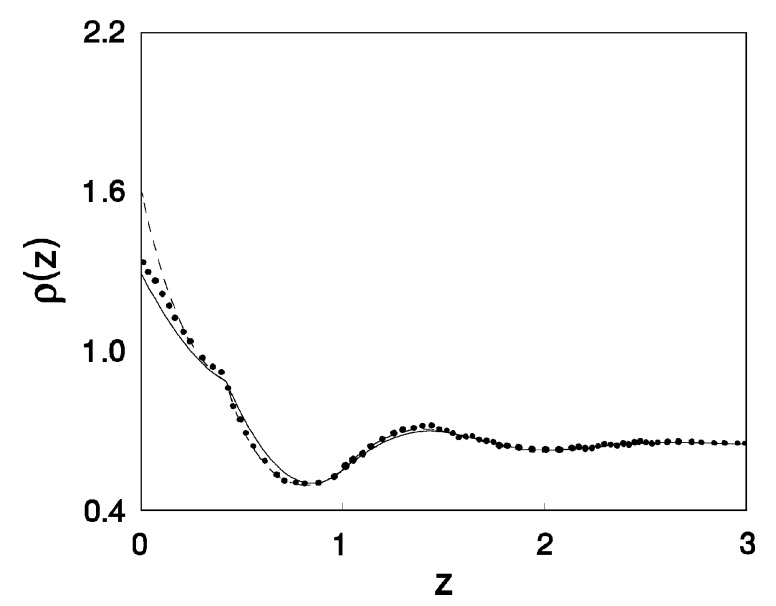

Fig. 1. The total local density $\rho(z)$ for $\rho=0.646$ and $\beta \varepsilon_{b}=4.25$. The solid line is for PY1/EMSA, the dashed line is for HNC1/EMSA, the squares are the MC simulation results [23].

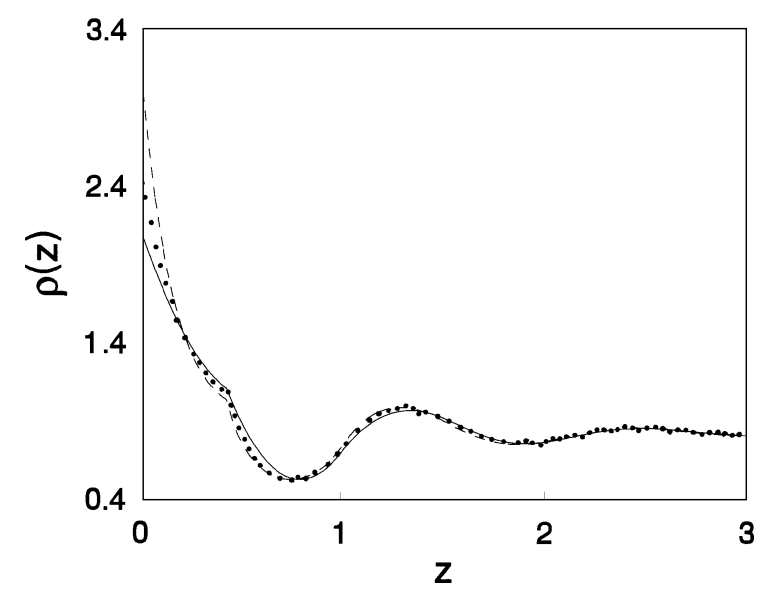

Fig. 2. The same as in Fig. 1 at $\rho=0.821$.

We have already discussed the effect of association on the DPs for this model in the PY1/PY and PY1/HNC approximations $[38,39]$. The contact value of the DPs is lower for an associated fluid compared to a hard sphere fluid. For the states of incomplete association monomers adsorb easier on the wall than the dimers. However, for a highly dimerized fluid, such as presented in Figs. 1 and 2 , the adlayer is mainly composed of the dimer species which prefer to lie parallel to the wall plane. This follows from the position of the cusp. One cannot discuss the lateral correlations in detail when only the DPs are available. It is worth noting that the simulation data show that some of the dimers are tilted with respect to the surface which results in a shift into the bulk of the position of the center of mass of the dimers [49]. In general, the correlations in the normal direction decay faster for the increasing dimerization. This effect, in particular, leads to a weak oscillation of the solvation force for the associated fluids in slit-like pores [50].

It is of special interest for various chemical problems to formulate a more sophisticated model for the bulk fluidsolid interactions. In particular, an interplay between the attractive and repulsive interactions would lead to the possibility of wetting. Here, we would like to focus on another issue, namely on the penetrability of the surface. Modelling of penetrability is important in different applications. For example, in experimental studies of colloidal systems, the surface of a colloid is often made by means of a chemical reaction at the interface [51]. In general, surface chemical reactions represent a novel fascinating subject of research.

On the other hand, even in the absence of a surface chemical reaction, the incorporation of certain bulk species into the composite penetrable interfacial region would lead to the behaviour of the solvation force between the solid surfaces which is similar to experiment [52]. As an initial step in the study of the effects of penetrability, we consider a simple model of a finite gaussian barrier for the particle-surface interactions [53] which has been used for the case of simple fluids [54]. It is chosen in the form of

$$
U_{m}(z)=h_{m}\left[1+\left(2 \omega^{-1}|z|\right)^{9}\right]^{-1}
$$

where $\omega$ is the halfwidth of the barrier located at $z=$ 0 . The parameter $h_{m}$ denotes the height of the barrier for the species $m$. The DPs for the model defined by Eqs. (2.5), (2.6) and (2.18) are calculated by using the HNC1/EMSA approximation. The HNC1 is used because it is successful for simple fluids in the presence of penetrable barriers [54]. The DPs are shown in Figs. 3 and 4 . We assume $h_{A}=h_{B}=h$ for simplicity and transparency of analysis and consider a dense $(\rho=0.57)$ highly dimerized fluid $\left(\beta \varepsilon_{b}=4.0, \Lambda_{b}=0.879\right.$ for $L_{b}=0.42$ ). In the case of a wide barrier (Fig. 3 , $\omega=1.42$ which coincides with dimer length) one observes a maximum of the DP at $z=0$ in spite of a maximum repulsion at the center of the barrier. However, the value of the DP at $z=0$ is higher for a hard sphere fluid, reflecting a higher penetrability of the monomeric units compared to the dimers. The first maximum of the DP outside the barrier is of the same order for the hard sphere and the highly associated fluid. The decay of correlations outside the barrier is somewhat faster for the highly dimerized fluid. With increasing strength of repulsion, the height of the local maximum at the center of barrier decreases. It decreases faster for a highly dimerized fluid. 

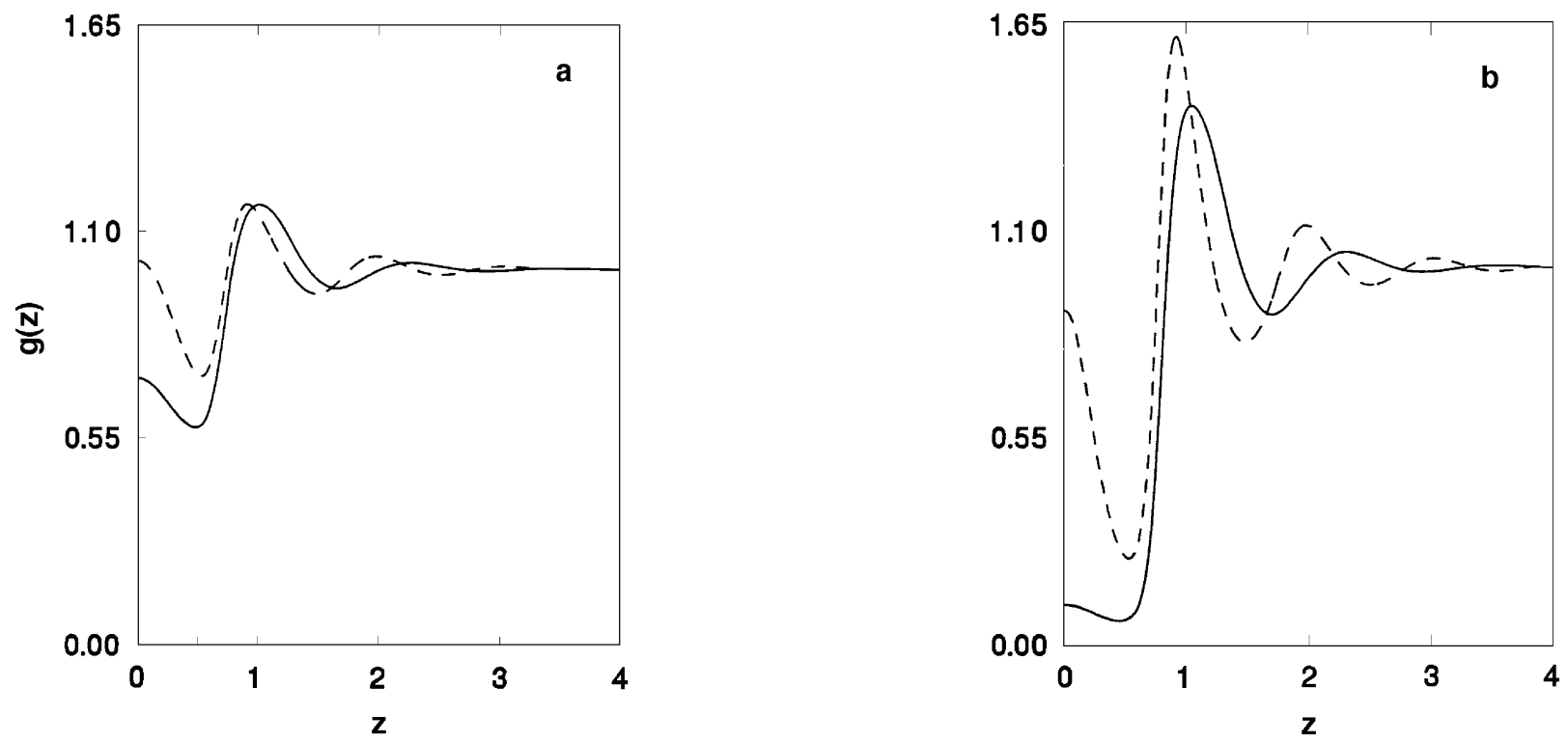

Fig. 3. Density profiles of particles inside and near the isolated barrier for hard spheres (dashed curves) and highly dimerized fluid (solid curves) for $\rho=0.57, \omega=1.42, h=1$ (a) and $h=4$ (b).
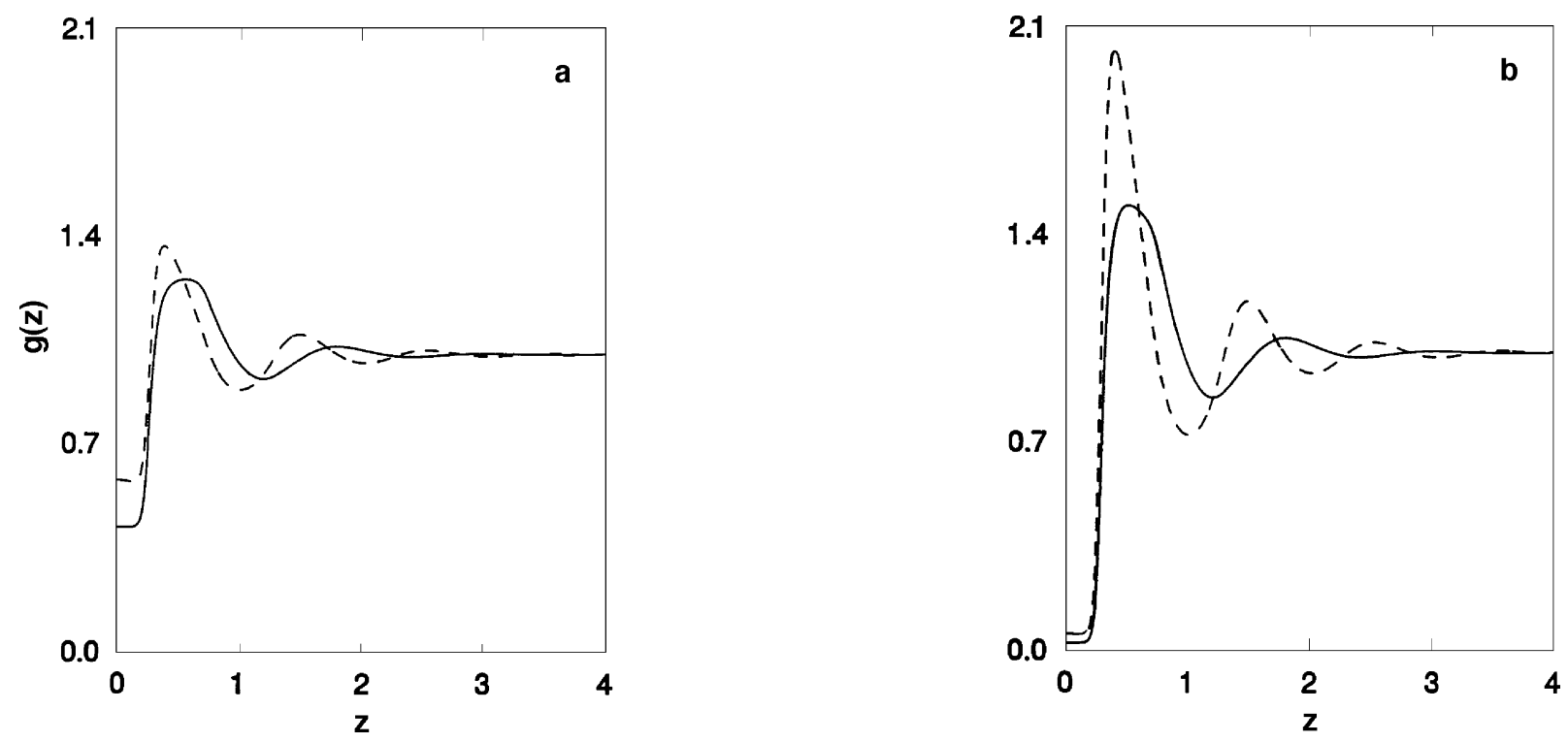

Fig. 4. Density profiles of particles inside and near the isolated barrier for hard spheres (dashed curves) and highly dimerized fluid (solid curves) for $\rho=0.57, \omega=0.5, h=1$ (a) and $h=4(\mathrm{~b})$.

For a narrow barrier $(\omega=0.5$, Fig. 4$)$, there is no local maximum of the density in the center of barrier. This local maximum arises only for barriers whose width is commensurate with the length of dimer species. Also a difference in the height of the first maximum of the DP outside barrier is observed for the hard sphere and the highly dimerized fluid. The dimers prefer to be adsorbed on the surface of the barrier and are mostly parallel to the surface. It is worth noting that there is no cusp on the DP, in contrast to the case of a hard wall. In general, the different penetrability of the monomeric and multimer species can be used to form adlayer with specific composition.

Now let us proceed and describe the DPs which follow from the application of Wertheim's theory for a model of homogeneous association near a hard wall defined by Eqs. (2.14)-(2.16). Actually here we pursue different goals. As it has been discussed in the introduction, a universal theory for the bulk associating fluids which is successful for a wide range of densities is not available. The 
DPs for the different models discussed above correspond to high densities. The two-density formalism developed within the framework of Wertheim's theory [5] is successful for low densities. In Figs. 5 and 6 we show the results for the DPs calculated in the PY1/APY approximation.

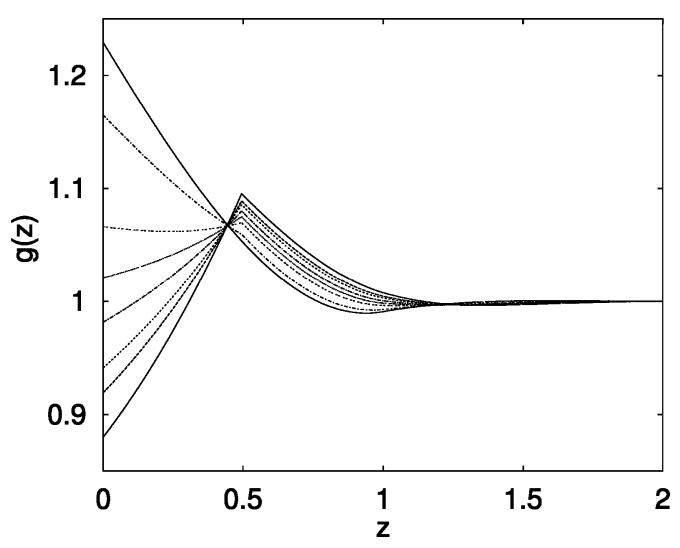

Fig. 5. The dependence of the normalized density profiles $g(z)$ on $K_{0 b}$. The bulk fluid density is $\rho=0.1, L_{b}=0.5$. The curves from the bottom at $z=0$ are for $K_{0 b}=1000,100,50$, $20,10,5,1$ and 0 .

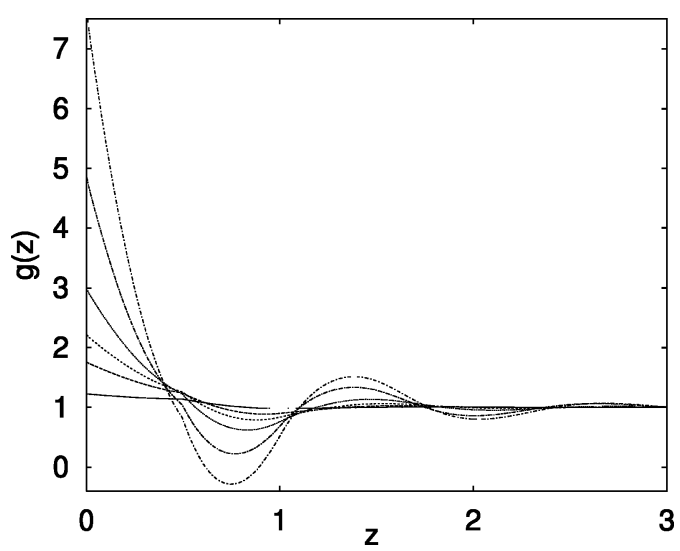

Fig. 6. The dependence of the normalized density profiles $g(z)$ on $\rho$ evaluated for $K_{0 b}=5, L_{b}=0.5$. The curves from the bottom at $z=0$ are for $\rho=0.2,0.4,0.5,0.6,0.7$ and 0.75 .

The dependence of the normalized DPs $g(z)$ on the strength of the associative interaction for the model with $L_{b}=0.5$ is presented in Fig. 5. Similarly to other associating fluids a small extent of the particle-wall correlations is observed. The cusp at $z=L_{b}$ indicates the presence of the dimerized species in the adlayer. An important effect worth discussing is the decrease of the contact value of the DPs for increasing level of dimerization. For a highly dimerized fluid at low density, the contact value of the DP on the wall can become less than unity, i.e. the so-called depletion of the profile is observed. For higher densities, a lowering of the contact value of the DP on the wall is obtained at increasing level of dimerization.
As expected, at fixed strength of association (fixed $K_{0 b}$ ), longer range of the fluid-wall correlation is observed for increasing density. For higher densities the packing effect prevails and the role of association is weaker. As before, association is manifested by the cusp at $L_{b}$ (Fig. 6), even in the presence of multilayer structure due to the packing effects. In general, the contact values for the DPs of the shorter dimers are lower compared with the case of longer dimers.

It is worth discussing briefly another important issue which results from the application of Wertheim's theory, that we had not realized previously. It is possible to treat the associating fluid-solid interactions in detail and distinguish between the effects of the bonded and nonbonded species in the contact adlayer and in the vicinity of the surface. This information is probably less important in thermodynamic aspect (for example, the adsorption isotherms resulting from both contributions). However, it gives an insight into the structure of the adlayer and its composition. It is possible to develop associative closures for the fluid--solid interactions which distinguish betweeen the bonded and nonbonded species and as a result one could discuss a number of dimers, for example, over a specific position on the surface.

\section{SINGLET LEVEL THEORY FOR DIMERIZING FLUIDS IN CONTACT WITH CRYSTALLINE SURFACES}

\section{A. General consideration}

In this section we use the basic equations for the density profiles, given by Eqs. (2.3), (2.4) using the PY1 and HNC1 approximation. However, with crystalline surfaces it is necessary to develop additional procedures. We focus on the PY1 equation because its transformation for crystalline surfaces is more straightforward and contains less technical details. The HNC1 implementation for crystalline surfaces is more cumbersome. It has been presented recently in our paper [40] and we omit that procedure for the sake of brevity. Consider nonassociative crystalline surfaces first, where there is no associative interaction between the fluid particles and atoms which constitute the solid subsystem. In this presentation we follow the method of Steele [55]. It is assumed that the summation of interactions between a given fluid particle and all the atoms of the solid leads to the formation of effective potentials between the fluid particle and solid as a whole. The potential energy of these interactions is given as

$$
U_{f s}^{p o t}=\sum_{m=A, B} \sum_{j} U_{m}\left(\mathbf{r}_{j}\right)
$$

The fluid-solid potentials $U_{m}\left(\mathbf{r}_{j}\right)$ are periodic functions in the surface plane. Thus $U_{m}\left(\mathbf{r}_{j}\right)=U_{m}\left(\mathbf{r}_{j}+\mathbf{l}\right)$, where $\mathbf{l}=l_{1} \mathbf{a}_{1}+l_{2} \mathbf{a}_{2}, \mathbf{a}_{1}, \mathbf{a}_{2}$, are two-dimensional unit lattice vectors and $l_{1}, l_{2}$ are integers. Following to Steele [55], the 
nonassociative interaction potential between the spherically symmetric particles of the bulk fluid and solid surface are given as

$$
\frac{1}{\varepsilon_{f s}} U_{m}\left(\mathbf{r}_{j}\right)=E_{m, 0}\left(z_{j}\right)+\chi \sum_{l>0} E_{m, l}\left(z_{j}\right) f_{l}\left(s_{1}, s_{2}\right)
$$

where the first term describes the interaction averaged over the solid atoms and the second term represents all the effects due to the crystalline symmetry of the surface. For all the details concerning these potentials we refer to Ref. [55]. The distance between the fluid particle and the surface is measured in dimensional units, i.e. in terms of the length of the unit lattice vector. The parameter $\chi$ can be used to magnify or decrease energetic effects of periodicity. The functions $f_{l}\left(s_{1}, s_{2}\right)$ have been given by Steele for some types of the crystalline lattices. We shall use also the estimates of Steele for the energy parameter $\varepsilon_{f s}$.

Because of the periodicity of the crystal surface plane, all the functions which characterize the position of the fluid particle over the surface can be expanded into a two-dimensional Fourier series

$$
\psi_{m}\left(\mathbf{r}_{j}\right)=\sum_{\mathbf{q}>\mathbf{0}} \psi_{m, \mathbf{q}}\left(z_{j}\right) \exp \left(-i \mathbf{q} \mathbf{t}_{j}\right)
$$

where $\psi_{m}\left(\mathbf{r}_{j}\right)$ stands for $y_{m}\left(\mathbf{r}_{j}\right)$ or $\gamma_{m}\left(\mathbf{r}_{j}\right)$ and $\rho_{m}\left(\mathbf{r}_{j}\right)$, as appropriate. The vector $\mathbf{t}$ is the two-dimensional translation vector in the plane parallel to the surface, $\mathbf{t}=s_{1} \mathbf{a}_{1}+s_{2} \mathbf{a}_{2}$ in which $s_{1}, s_{2}$ range from 0 to 1 as the position of a fluid particle moves over a unit surface cell. The vector $\mathbf{q}$ is the reciprocal lattice vector, $\mathbf{q}=2 \pi\left(q_{1} \mathbf{b}_{1}+q_{2} \mathbf{b}_{2}\right)$, where $\mathbf{b}_{1}, \mathbf{b}_{2}$ are two-dimensional reciprocal lattice unit vectors and $q_{1}, q_{2}$ are integers. Inverse transformation of Eq. (3.3) yields the expansion coefficients

$$
\psi_{m, \mathbf{q}}\left(z_{j}\right)=a_{s}^{-1} \int d \mathbf{t}_{j} e^{i \mathbf{q t}_{j}} \psi_{m}\left(\mathbf{r}_{j}\right)
$$

where $a_{s}$ is the area of the unit lattice cell. Using the transformation (21), the PY1 equation is rewritten in the form

$$
\begin{aligned}
& y_{m, \mathbf{q}}\left(z_{i}\right)=a_{m}^{*} \delta_{\mathbf{q}, 0}+ \\
& \sum_{\mathbf{q}_{1}+\mathbf{q}_{2}=\mathbf{q}} \sum_{n=A, B} \rho_{n} \int d z_{j} y_{n, \mathbf{q}_{2}}\left(z_{j}\right) \gamma_{n, \mathbf{q}_{1}}\left(z_{j}\right) c_{m n}\left(z_{i j},|\mathbf{q}|\right),
\end{aligned}
$$

where

$$
a_{m}^{*}=1-2 \pi \sum_{n=A, B} \rho_{n} \int d r r^{2} c_{m n}(r)
$$

and

$$
\begin{aligned}
& c_{m n}\left(z_{i j}, q\right) \\
& =2 \pi \sum_{n=A, B} \rho_{n} \int d r r J_{0}(q r) c_{m n}\left(\left[z_{i j}^{2}+r^{2}\right]^{1 / 2}\right),
\end{aligned}
$$

and where $J_{0}(q r)$ is the Bessel function of the first kind. Due to the properties of the direct and reciprocal lattices Eq. (3.5) can be made more simple. The expansion coefficients in Eq. (3.3) depend only on the magnitude of the vector $\mathbf{q}$. Therefore the expansion for the cavity function $y_{m}(z)$ can be written in the form of a series, ordered by the increasing value of the wave vector. The expansion for the cavity function can be presented in the form [40, $41,56]$

$$
y_{m}(\mathbf{r})=y_{m, 0}(z)+\sum_{l \geq 1} y_{m, l}(z) f_{l}\left(s_{1}, s_{2}\right)
$$

where the functions $f_{l}\left(s_{1}, s_{2}\right)$ have been given in Ref. [55] for (100) and (111) fcc lattice planes and for graphite basal plane. In what follows we shall present some of the results for (100) lattice plane. For the technical details of the calculations and the explicit form of particle-solid interactions see Refs. [40, 41, 55].

\section{B. Density profiles}

The DPs are presented for the model of heterogeneous dimerization due to Cummings and Stell [4]. The fluid is in contact with the (100) solid surface. For simplicity, we restrict our attention to the case where the bulk fluid particles and solid atoms are of equal size and $L_{b}=0.5$. The DPs were calculated by using the PY1/PY approximation and are shown in Figs. 7 and 8. We assumed that the interactions of both the bulk species with the surface are identical, Therefore the DPs of each species are equal. The effect of a strong fluid-solid interaction and that of dimerization can be discussed using the DPs shown in Fig. 7. For a small degree of dimerization and a relatively low packing fraction (see the figure caption) the structure of the adlayer is different from that for a high degree of dimerization. The effect of dimerization leads to a decrease in the height of the first maximum of the DP over the adsorbing site position $s$ of the unit lattice cell. The dimers prefer to adsorb over this position compared with the saddle point $s p$ and atom $a$ positions. The dimers prefer to be normal to the surface plane, in contrast to the case of a dimerized fluid in contact with a hard wall. However, as follows from the increase of the first maxima over the $s p$ and $a$ positions for increasing dimerization, the tilted orientations of dimers adsorbed in the $s$ position are also probable. The normal correlations decay rapidly at this density and a multilayer structure of the adlayer is not observed. The effect of the periodicity of the crystalline lattice plays an important role on the shape of the DPs (see Fig. 8). It is evident that an increase of the strength of periodicity of the fluid-solid 
interactions leads to the expulsion of the dimer species from the atom positions. The dimerized particles prefer to be adsorbed over the $s$ and $s p$ positions of the unit lattice cell. The effect of a high packing fraction results in the formation of the structure which is different from that for a hard sphere fluid.

In all the studies discussed above only the nonassociative adsorption of bulk species on the crystalline surfaces has been considered. Localization of the species in the adlayer is generated solely by nonassociative interactions between the fluid particles and the atoms of the surface. A more sophisticated model which includes associative adsorption on the crystalline lattices has been proposed recently in Ref. [57]. We refer to this model as to the model for localized site adsorption. Associative adsorption is characterized by a high degree of localization of adsorbed monomers and dimers as a consequence of a strong bonding between the bulk species and solid atoms. In this model the interaction between the bulk fluid particles and solid is taken in the form

$$
U_{m}(\mathbf{r})=U_{m}(z)+U_{m}^{(a)}(\mathbf{r})
$$

where the first term is chosen as a hard wall potential whereas the second is given as

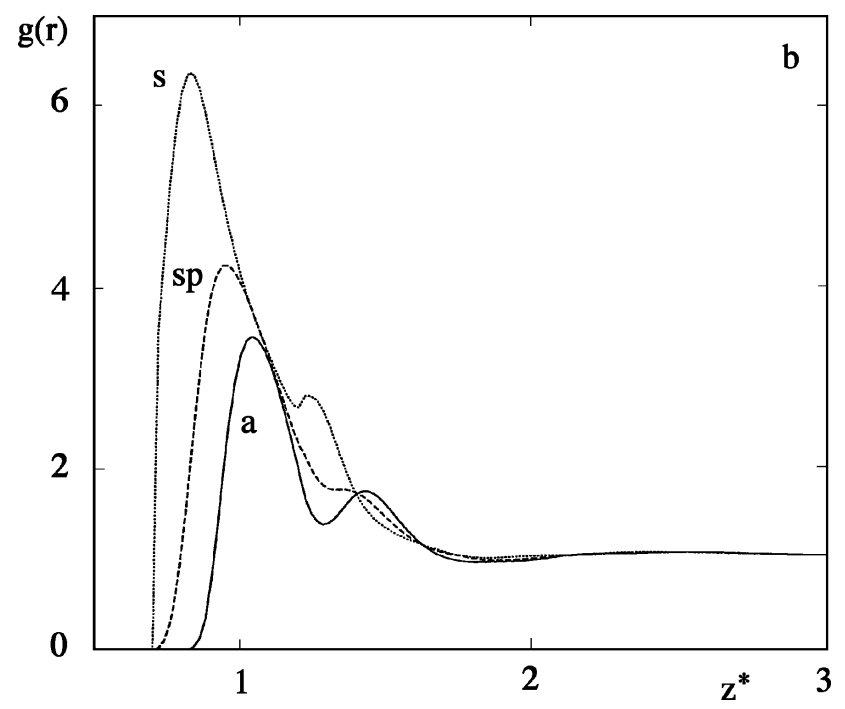

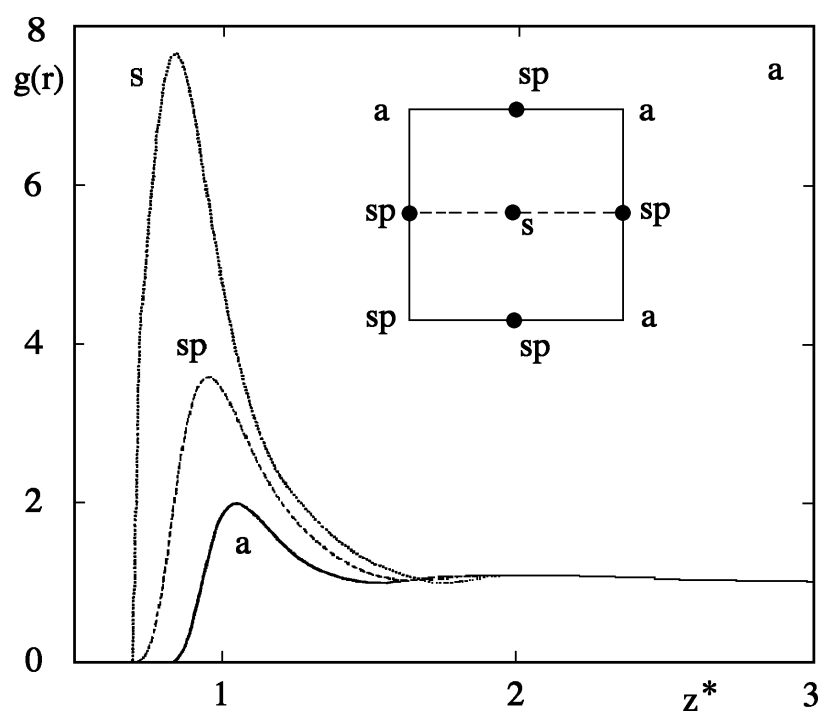

Fig. 7. The normalized density profiles $g(z)$ as a function of distance from the surface obtained for $\beta \varepsilon_{f s}=0.25, \chi=1$, $\rho=0.191 L_{b}=0.5$ and for $K_{0 b}=0.131$, weak dimerization (part a) and for $K_{0 b}=13.1$, high dimerization (part b). The labels $\mathrm{s}, \mathrm{sp}$ and a denote the (s), (sp) and (a) positions, respectively. The inset in part a shows the surface lattice unit cell and the location of the (s), (sp) and (a) positions.
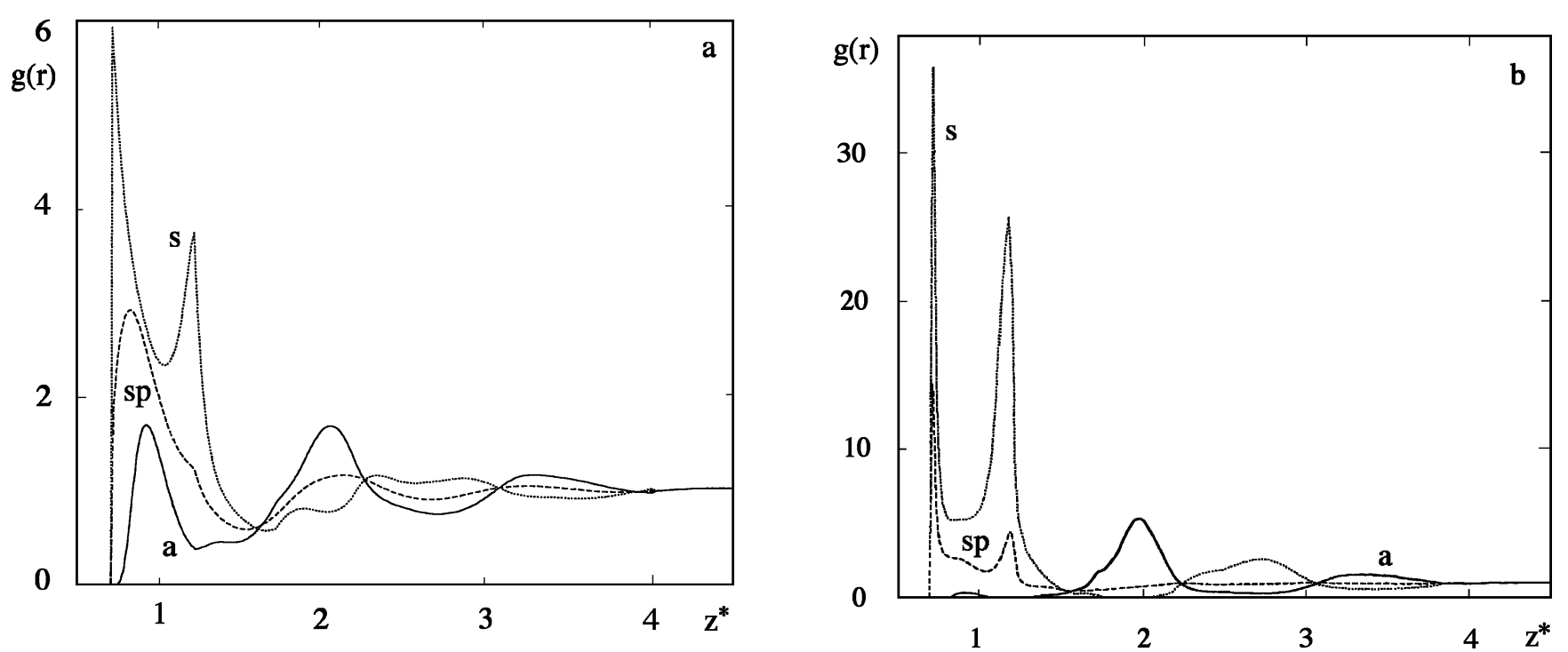

Fig. 8. The normalized density profiles $g(z)$ obtained for $\beta \varepsilon_{f s}=0.05, \rho=0.764, K_{0 b}=13.1$, and for $\chi=1$ (part a) and $\chi=2$ (part b). The nomenclature of the lines is as in Fig. 7 . 


$$
U_{m}^{(a)}(\mathbf{r})=\delta_{m A}\left\{\begin{array}{cc}
\infty, & |\mathbf{r}|<L_{s}-0.5 w \\
-\varepsilon_{s}, & L_{s}-0.5 w<|\mathbf{r}|<L_{s}+0.5 w \\
\varepsilon_{m}, & L_{s}+0.5 w<|\mathbf{r}|<1 \\
0, & |\mathbf{r}|>1
\end{array},\right.
$$

where $L_{s}$ is the bonding distance for the fluid-solid interaction and $\delta_{i j}$ denotes the Kronecker symbol. The other notation coincides with those for the model of Cummings and Stell [4]. The strength of the fluid-solid energy is given by the energy parameter $\varepsilon_{s}$. The potential $U_{m}^{(a)}(\mathbf{r})$ is a periodic function in the crystal plane, i.e. $U_{m}^{(a)}(\mathbf{r})=U_{m}^{(a)}(\mathbf{r}+\mathbf{l})$, where $\mathbf{l}$ is the two-dimensional translation vector. The parameter $L_{s}$ determines the type of the complexes of the bulk fluid particles and surface atoms which are formed. In the following calculations we have used the SSEMSA approach to describe the bulk model of Cummings and Stell for the heterogeneous asssociation [4] and the PY1 approximation to evaluate the profiles. The surface atoms in what follows will be denoted as $S_{a}$.

The results for the DPs are presented in Figs. 9-11.
All correspond to $\beta \varepsilon_{s}=2.0$ and the width of the square well $w=0.05$. The bulk model is taken at the density $\rho=0.668$ and $L_{b}=0.475$ and for $\beta \varepsilon_{b}=1.0$ and $\beta \varepsilon_{b}=3.0$. These values for the bulk bonding energy provide a highly dimerized fluid with $\Lambda_{b}=0.559$ and $\Lambda_{b}=0.784$ respectively. In Fig. 9 we present the case of a (100) lattice plane and different bonding lengths, $L_{s}$. For small $L_{s}\left(L_{s}=0.425\right.$ and $\beta \varepsilon_{b}=3.0$, Fig. 9a), an intraparticle peak over atom position which corresponds to $A-S_{a}$ bonding is observed. Such adsorption is usually called on-top localized site adsorption. However, the monomer $A$ can be tilted with respect to the normal to the surface plane. The dimers $A B$ cannot adsorb associatively due to the chosen values of the bonding length. Two intraparticle peaks arise for a larger bonding distance $L_{s}\left(L_{s}=0.65\right.$ and $\beta \varepsilon_{b}=1.0$, Fig. 9b). In this case we identify them as $A-S_{a}, B A-S_{a}$, and $A-2 S_{a}$, $B A-2 S_{a}$ bonding. The first intraparticle peak corresponds to the on-top monomer and on-top dimer adsorption whereas the second (over $s p$ position) corresponds to the two-fold bridging site adsorption of the monomer $A$ and dimer $A B$. It is worth noting that for small bonding distances the species profiles do not differ by the interparticle terms and, therefore, we present the profiles for $A$ particles only. In Fig. 9c three intraparticle peaks are present for $L_{s}=0.9\left(\beta \varepsilon_{b}=3.0\right)$. The intraparticle peak
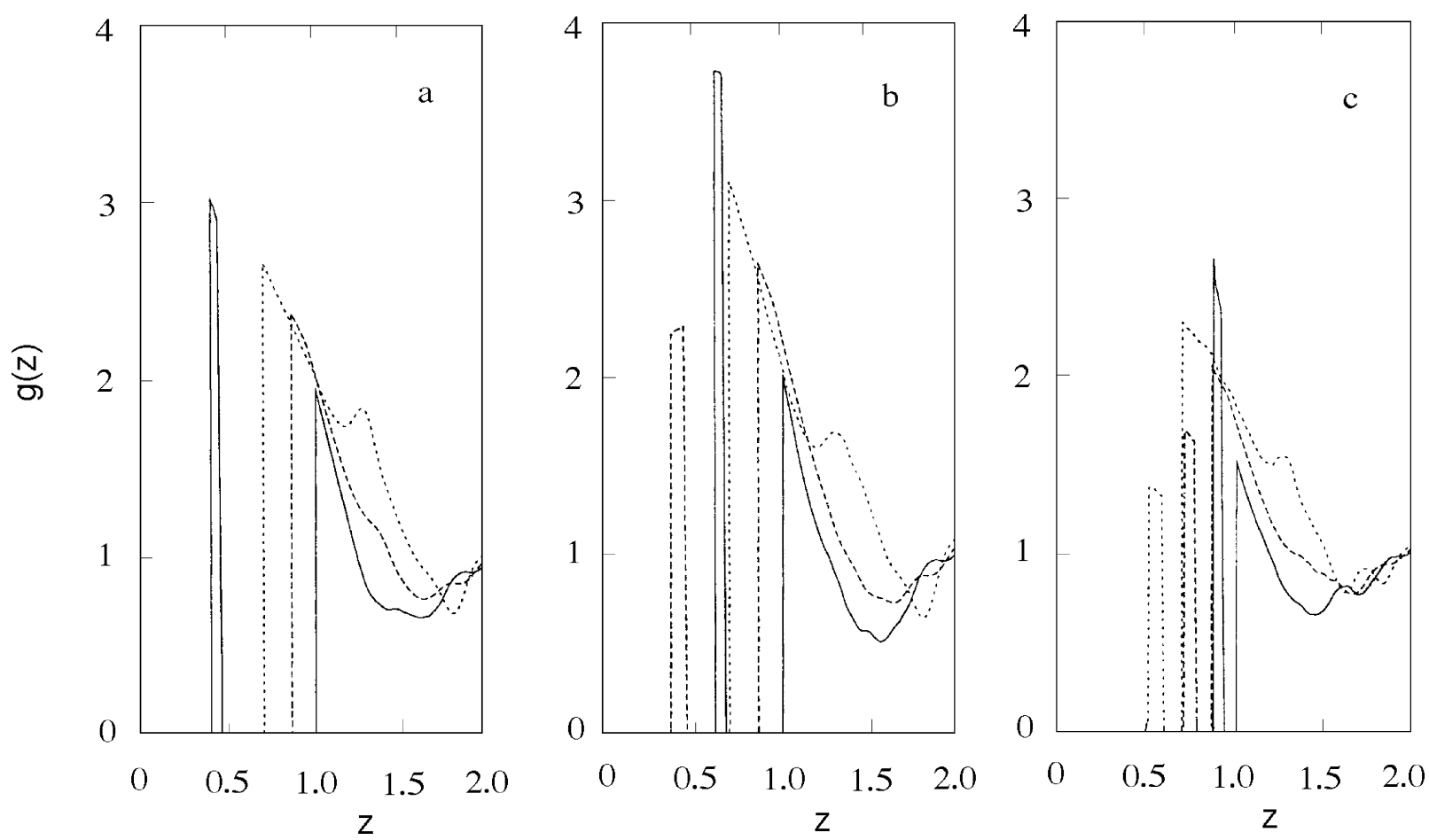

Fig. 9. Density profiles of $A$ particles over the unit lattice cell of (100) surface. Nomenclature of the lines is the following: $a$ position - solid line, $s p$ - dashed line, $s$ - short dashed line. Part $\mathbf{a}$ is for $\beta \varepsilon_{b}=3$ and $L_{s}=0.425$ (the intraparticle peak is multiplied by 0.5 ). Part $\mathbf{b}$ is for $\beta \varepsilon_{b}=1$ and $L_{s}=0.65$ (the intraparticle peak at $s p$ position is multiplied by 0.1 and at $a$ position is scaled as previously). Part $\mathbf{c}$ is for $\beta \varepsilon_{b}=3$ and $L_{s}=0.9$ (the intraparticle peak over $s$ position is multiplied by 0.01 whereas over the $s p$ position and $a$ position are scaled as previously). In Figs. $9-11 L_{b}=0.475$. 

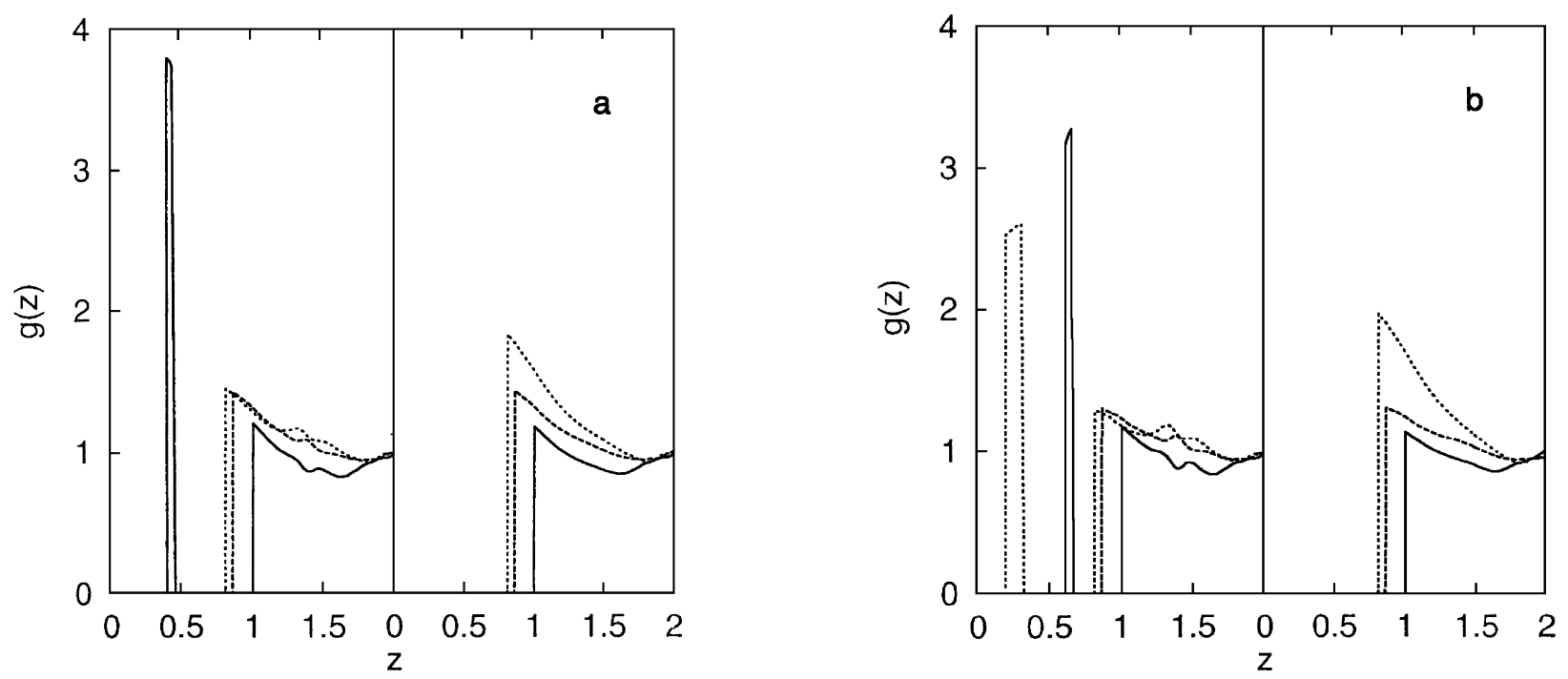

Fig. 10. Density profiles of $A$ and $B$ particles over the unit lattice cell of (100) surface (left and right panel, respectively) for $\beta \varepsilon_{b}=1$ and $L_{s}=0.9$. Nomenclature of the lines is as in Fig. 9 (the intraparticle peaks are scaled as in Fig. 9c).

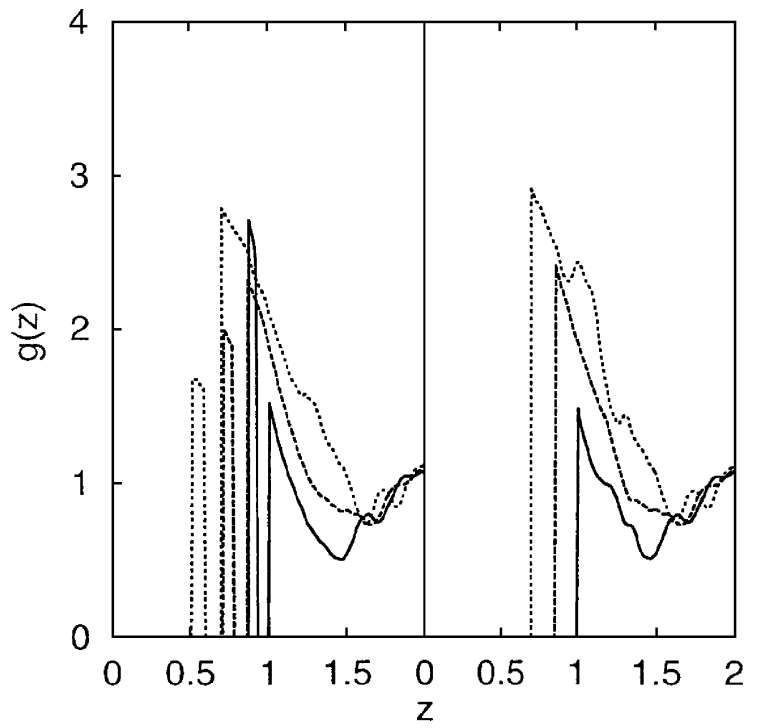

Fig. 11. Density profiles of $A$ and $B$ particles over the unit lattice cell of (111) surface plane (left and right panels in each part, respectively) for $\beta \varepsilon_{b}=1$ and $L_{s}=0.425$ (part a) and for $\beta \varepsilon_{b}=3$ and $L_{s}=0.635$ (part $\mathbf{b}$ ). Nomenclature of the lines is as in Fig. 9.

over the $s$ position corresponds to $A-4 S_{a}, B A-4 S_{a}$ bonding which are called the four-fold hollow site adsorption of the monomer and dimer. In all the cases decribed above, it could be seen that nonassociatively adsorbed dimers prefer to locate over the $s$ position of the unit lattice cell. In general, the range of normal correlations is not larger than the dimer length. A higher association of the bulk fluid (cf. Fig. 9c and Fig. 10, the latter is for $L_{s}=0.9$ and $\beta \varepsilon_{b}=1.0$, (100) lattice plane) leads to lower values of the intraparticle peaks, especially for $s$ and $s p$ positions in which associative adsorption is quite strong. An overall decrease in the contact values for the interparticle terms is observed too. For high values of the bonding distance, $L_{s}$, the profiles for $A$ and $B$ species differ by the interparticle terms. They differ because the surface complexes in this case are more exposed into the bulk. However, it is seen that the dimers prefer to orient normally to the surface plane.

Similar trends in the behaviour of the bulk model in contact with the (111) surface are observed by inspecting Fig. 11. In general, the (111) surface is more smooth energetically, as is evident from the values of the interparticle terms of the profiles. As noted previously, only one intraparticle peak is present for a small value of the bonding distance $\left(L_{s}=0.425\right.$ and $\beta \varepsilon_{b}=1.0$, Fig. 11a) whereas for larger $L_{s}\left(L_{s}=0.635\right.$ and $\beta \varepsilon_{b}=3.0$, Fig. 11b) two intraparticle peaks arise. They correspond to the on-top and two-fold bridging adsorption respectively. It is worth noting, that for (111) and graphite basal plane lattices, the complexes which involve three surface atoms can be obtained for the large values of the bonding length $L_{s}$. Our results are preliminary. A detailed investigation of all the possibilities for adsorption on the low-index lattices is postponed for future publications.

\section{PAIR LEVEL THEORY FOR INHOMOGENEOUS ASSOCIATING FLUIDS}

We start with general remarks. The PLT yields a much more detailed description of interfacial phenomena. It provides the inhomogeneous pair correlation functions as well as the density profiles of particles, in contrast to the SLT in which only the density profiles are given. Moreover, the PLT is able to describe surface phase transformations including the formation of adsorbate phases and wetting phase transitions.

The pair level theory consists of inhomogeneous OZ equation $(\mathrm{OZ2})$

$$
h_{i j}\left(\mathbf{r}_{1}, \mathbf{r}_{2}\right)-c_{i j}\left(\mathbf{r}_{1}, \mathbf{r}_{2}\right)
$$




$$
=\sum_{n} \int d \mathbf{r}_{3} h_{a n}\left(\mathbf{r}_{1}, \mathbf{r}_{3}\right) c_{n b}\left(\mathbf{r}_{3}, \mathbf{r}_{2}\right) \rho_{n}\left(\mathbf{r}_{3}\right)
$$

and additional equation for the density profiles such as the Lovett-Mou-Buff-Wertheim equation,

$$
\nabla \ln y_{i}\left(\mathbf{r}_{1}\right)=\sum_{n} \int d \mathbf{r}_{2}\left[\nabla \rho_{n}\left(\mathbf{r}_{2}\right)\right] c_{i n}\left(\mathbf{r}_{1}, \mathbf{r}_{2}\right)
$$

where $y(\mathbf{r})$ is the one-particle cavity distribution function. Other equations for the profile, such as the BornGreen-Yvon equation, can be used instead of Eq. (4.2). To make the problem complete one must supplement Eqs. (4.1) and (4.2) by a closure relation for the inhomogeneous direct correlation function.

In most previous studies of inhomogeneous simple fluids, by means of PLT, the inhomogeneous Percus-Yevick (PY2) approximation has been used [37]. It reads

$$
c_{i j}\left(\mathbf{r}_{1}, \mathbf{r}_{2}\right)=y_{i j}\left(\mathbf{r}_{1}, \mathbf{r}_{2}\right) f_{i j}\left(r_{12}\right),
$$

where $y_{i j}\left(\mathbf{r}_{1}, \mathbf{r}_{2}\right)$ is the inhomogeneous cavity pair distribution function and $f_{i j}\left(r_{12}\right)$ is the Mayer function corresponding to the pair interparticle interaction. The inhomogeneous hypernetted chain closure (HNC2)

$$
\begin{aligned}
& c_{i j}\left(\mathbf{r}_{1}, \mathbf{r}_{2}\right)=-1 \\
& +y_{i j}\left(\mathbf{r}_{1}, \mathbf{r}_{2}\right)\left[f_{i j}\left(r_{12}\right)+1\right]-\ln \left[y_{i j}\left(\mathbf{r}_{1}, \mathbf{r}_{2}\right)\right],
\end{aligned}
$$

can be used too [37]. The HNC2 approximation is equivalent to setting the bridge function $B\left(\mathbf{r}_{1}, \mathbf{r}_{2}\right)$, equal to zero.

We would like to emphasize two important issues. First, it is clear that the implementation of the PLT is a completely numerical procedure. It is a complicated numerical task even for simple fluids. The presence of associative interactions makes the problem even more difficult technically. Secondly, the accuracy of the results depends on the closures applied. In this context we recall that usual liquid state closures, when applied for the bulk associating fluids, lead to deviations from the law of mass action. At distances far from the source of the inhomogeneity (a hard wall for example), the inhomogeneous correlation functions reduce to their bulk counterparts. Therefore, one needs to seek for the closures which for example reduce to the bulk EMSA closure for distances far from the wall.

Before considering this issue in more detail we discuss briefly some of the results obtained in Ref. [46] by using the PY2 approximation for the model of Cummings and Stell [4] in the one-dimensional external field

$$
U_{A}(z)=U_{B}(z)=\left\{\begin{array}{cc}
\infty, & z \leq 0 \\
0, & z>0
\end{array} .\right.
$$

The density profiles of the particles are shown in
Figs. 12 and 13. Obviously the PY2 results are in better agreement with the MC simulation data than the results of the SLT PY1. This improvement is observed at high density $(\rho=0.728)$ as well as at lower density $(\rho=0.391)$. Unfortunately, the interesting region

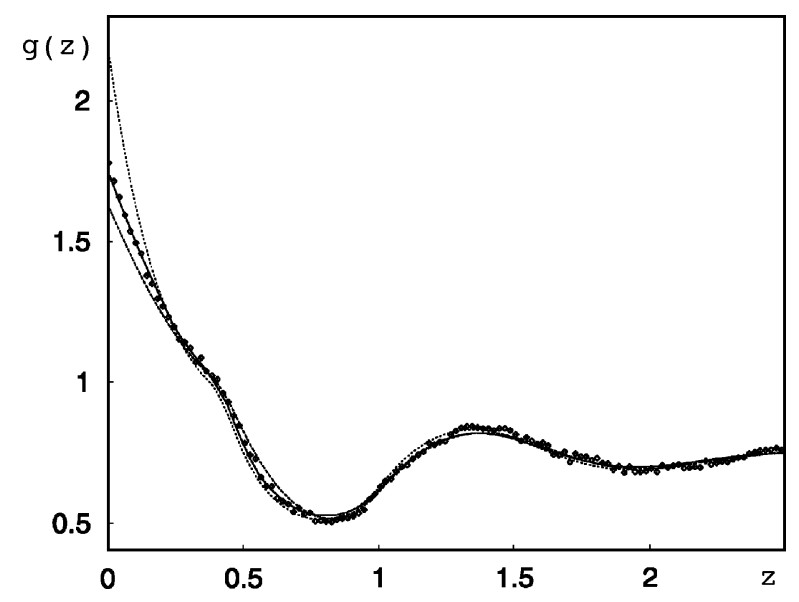

Fig. 12. A comparison of the MC (points), NPY (solid lines), HNC1 (short dashed lines), and PY1 (long dashed lines) density profiles. The model parameters used in calculations are: $\beta \varepsilon_{b}=4.245, L_{b}=0.42$, and $w=0.1$. The bulk density $\rho=0.728$.

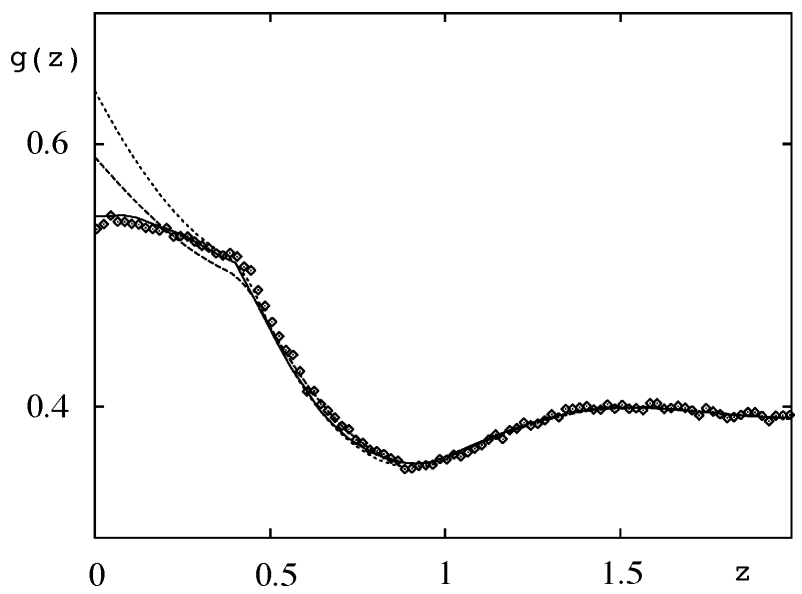

Fig. 13. The same as in Fig. 12 but the bulk density is $\rho=0.391$.

of very low densities as well as the dependence of the results on the strength of associative interaction have not been studied. Therefore, it is hard to judge the overall accuracy of the PY2 and the possibility of its use for a wide range of parameters. As expected, at large distances from the wall the profiles obtained by means of PY2 coincide with the PY1 results. The PY2 approximation is not completely satisfactory and we do not have an easy answer to the question: what closure is necessary in order to improve the results at distances far from the wall. One possibility would be to extend the PLT for IAFs in a manner similar to the application of a perturbative 
treatment which has been developed in another context in Ref. [58].

Let us assume that we have the results of the EMSA or SSEMSA theory for the bulk model which agree well with the simulation data, at least for a certain range of the parameters. These results can be used for the description of the reference fluid and the inhomogeneous closures can be used only for some part of the correlation functions. The inhomogeneous correlation functions can be written in the form

$$
\begin{aligned}
& h_{i j}\left(\mathbf{r}_{1}, \mathbf{r}_{2}\right)=h_{i j}^{r e f}\left(r_{12}\right)+\Delta h_{i j}\left(\mathbf{r}_{1}, \mathbf{r}_{2}\right), \\
& c_{i j}\left(\mathbf{r}_{1}, \mathbf{r}_{2}\right)=c_{i j}^{r e f}\left(r_{12}\right)+\Delta c_{i j}\left(\mathbf{r}_{1}, \mathbf{r}_{2}\right),
\end{aligned}
$$

where $h_{i j}^{r e f}\left(r_{12}\right), c_{i j}^{r e f}\left(r_{12}\right)$ stand for the correlation functions of the bulk associating fluid. It is not necessary to assume any particular approximation for the bulk fluid. We assume that the choice of the reference fluid description is successful. The functions $\Delta f_{i j}(f \Rightarrow(h, c))$ are the deviations of the corresponding inhomogeneous correlation functions from their bulk counterparts. They are significant only in the range of the inhomogeneity. The correlation functions of the reference fluid satisfy the OZ equation:

$$
h_{i j}\left(r_{12}\right)-c_{i j}\left(r_{12}\right)=\sum_{n} \int d \mathbf{r}_{3} h_{i n}\left(r_{13}\right) c_{n j}\left(r_{32}\right)
$$

Let us obtain now the difference OZ2 (DOZ2) integral equation for the functions $\Delta f_{i j}(1,2)=\Delta f_{i j}\left(\mathbf{r}_{1}, \mathbf{r}_{2}\right)$ by subtracting the OZ from the OZ2 equation. The DOZ2 equation has the form:

$$
\begin{gathered}
\Delta h_{i j}(1,2)-\Delta c_{i j}(1,2)=\sum_{n}\left\{\int d \mathbf{r}_{3}\left(\rho_{n}\left(\mathbf{r}_{3}\right)-\rho_{n}\right) h_{i n}^{r e f}(13) c_{n j}^{r e f}(32)\right. \\
\left.+\int d \mathbf{r}_{3} \rho_{n}\left(\mathbf{r}_{3}\right)\left[\Delta h_{i n}(1,3)\left(c_{n j}^{r e f}(32)+\Delta c_{n j}(3,2)\right)+h_{i n}^{r e f}(13) \Delta c_{n j}(3,2)\right]\right\} .
\end{gathered}
$$

Before considering the integral equation for the profile, we proceed directly to the closure relations. To derive them, consider the exponential representation for the inhomogeneous pair distribution function:

$$
\begin{gathered}
g_{i j}(1,2)=\exp \left\{-\beta U_{i j}(12)+h_{i j}(1,2)-c_{i j}(1,2)+B_{i j}(1,2)\right\} \\
=g_{i j}(12) \exp \left\{\Delta h_{i j}(1,2)-\Delta c_{i j}(1,2)+\Delta B_{i j}(1,2)\right\}
\end{gathered}
$$

where $g_{i j}(12)$ is the exact pair distribution function of the bulk fluid (an associating fluid in our case). This $g_{i j}(12)$ corresponds to the reference fluid. The difference HNC2 (DHNC2) closure is defined in a standard way:

$$
\Delta B_{i j}(1,2)=0
$$

where $\Delta B_{i j}(1,2)$ is the difference in the bridge function. Equation (38) leads to

$$
\Delta c_{i j}(1,2)=\Delta h_{i j}(1,2)-\ln \left\{1+\frac{\Delta h_{i j}(1,2)}{g_{i j}^{r e f}(12)}\right\} .
$$

The difference PY2 (DPY2) approximation is obtained by the linearization of the logarithmic term in Eq. (4.11)

$$
\Delta c_{i j}(1,2)=\Delta h_{i j}(1,2) \frac{h_{i j}^{r e f}(12)}{g_{i j}^{r e f}(12)}
$$

Now we can rewrite the final form of the DOZ2 equation in the DPY2 approximation

$$
\begin{gathered}
\Delta h_{i j}(1,2)-\Delta c_{i j}(1,2)=\sum_{n}\left\{\int d \mathbf{r}_{3}\left(\rho_{n}\left(\mathbf{r}_{3}\right)-\rho_{n}\right) h_{i n}^{r e f}(13) c_{n j}^{r e f}(32)\right. \\
\left.+\int d \mathbf{r}_{3} \rho_{n}\left(\mathbf{r}_{3}\right)\left[\Delta h_{i n}(1,3)\left(c_{n j}^{r e f}(32)+\Delta h_{n j}(3,2) \frac{h_{n j}^{r e f}(32)}{g_{n j}^{r e f}(32)}\right)+h_{i n}^{r e f}(13) \Delta h_{n j}(3,2) \frac{h_{n j}^{r e f}(32)}{g_{n j}^{r e f}(32)}\right]\right\}
\end{gathered}
$$

which is characterized by a transparent and quite simple nonlinearity on $\Delta h_{m n}(i, j)$. The DOZ2 equation within the 
DHNC2 closure has a more complicated form. We shall rewrite Eq. (4.13) in a form which is appropriate for the numerical procedure. Introduce the function:

$$
\Delta \gamma_{i j}(1,2)=\Delta h_{i j}(1,2)-\Delta c_{i j}(1,2)
$$

Then the DPY2 has a very convenient form

$$
\Delta c_{i j}(1,2)=\Delta \gamma_{i j}(1,2) h_{i j}^{r e f}(12)
$$

and the DOZ2 in the DPY2 approximation is given in a form which is convenient for numerical studies

$$
\begin{gathered}
\Delta \gamma_{i j}(1,2)=\sum_{n}\left\{\int d \mathbf{r}_{3}\left(\rho_{n}\left(\mathbf{r}_{3}\right)-\rho_{n}\right) h_{i n}^{r e f}(13) c_{n j}^{r e f}(32)+\int d \mathbf{r}_{3} \rho_{n}\left(\mathbf{r}_{3}\right)\left[( \Delta \gamma _ { i n } ( 1 , 3 ) + \Delta c _ { i n } ( 1 , 3 ) ) \left(c_{n j}^{r e f}(32)\right.\right.\right. \\
\left.\left.\left.+\Delta \gamma_{n j}(3,2) h_{n j}^{r e f}(32)\right)+h_{i n}^{r e f}(13) \Delta \gamma_{n j}(3,2) h_{n j}^{r e f}(32)\right]\right\}
\end{gathered}
$$

Let us now proceed with the equation for the profile. The LMBW equation is

$$
\nabla \ln y_{i}\left(\mathbf{r}_{1}\right)=\sum_{n} \sum \int d \mathbf{r}_{2}\left[\nabla \rho_{n}\left(\mathbf{r}_{2}\right)\right]\left[c_{\text {in }}^{r e f}(12)+\Delta \gamma_{i n}(1,2) h_{i n}^{r e f}(12)\right]
$$

Thus, the problem is complete. If one would like to focus on the description of the surface phase transitions it is possible to develop a more sophisticated closure which includes the bridge diagrams contribution. For any case a major part of the associative interaction is treated adequately by the introduction of the reference fluid. Any possible inaccuracies in the treament of bonding arise from the DPY2 approximation. We hope to investigate the scheme presented above in future studies.

\section{ACKNOWLEDGEMENT}

D.H. is grateful to CONACYT of Mexico (Grant No.4186-E9405) for partial financial support of this project. S.S. acknowledges support of KBN of Poland. O.P. is grateful to M.C.Lozada Garcia for valuable discussions and continuous interest in this project.

[1] H. C. Andersen, J. Chem. Phys. 59, 4714 (1973).

[2] D. Chandler, L. R. Pratt, J. Chem. Phys. 65, 2925 (1976); L. R. Pratt, D. Chandler, J. Chem. Phys. 66, 147 (1977).

[3] J. S. Høye, K. Olaussen, Physica A 104, 435 (1980).

[4] P. T. Cummings, G. Stell, Mol. Phys. 51, 253 (1984).

[5] M. S. Wertheim, J. Stat. Phys. 35, 19; 35 (1984).

[6] P. T. Cummings, G. Stell, Mol. Phys. 55, 33 (1985).

[7] P. T. Cummings, G. Stell, Mol. Phys. 60, 1315 (1987).

[8] S. H. Lee, P. T. Cummings, G. Stell, Mol. Phys. 62, $65(1987)$.

[9] S. H. Lee, J. C. Rasaiah, P. T. Cummings, J. Chem. Phys. 83, 317 (1985).

[10] J. C. Rasaiah, S. H. Lee, J. Chem. Phys. 83, 5870 (1985).

[11] J. C. Rasaiah, S. H. Lee, J. Chem. Phys. 83, 6396 (1985).

[12] J. C. Rasaiah, J. Zhu, J. Chem. Phys. 92, 7554 (1990).

[13] O. Pizio, J. Chem. Phys. 100, 548 (1994).

[14] O. Pizio, L. Blum, Phys. Rev. E 52, 572 (1995).

[15] G. Stell, Y. Zhou, J. Chem. Phys. 91, 3618 (1989).

[16] Y. Zhou, G. Stell, J. Chem. Phys. 96, 1504; 1507 (1992).

[17] Y. Zhou, G. Stell, J. Chem. Phys. 102, 2448 (1995).

[18] G. Stell, Y. Zhou, J. Chem. Phys. 91, 4861; 4869 (1989); Y. Zhou, H. L. Friedman, G. Stell, J. Chem. Phys. 91,
4879; 4885 (1989).

[19] G. Stell, Cond. Matt. Phys. (Lviv) 2, 4 (1993).

[20] Yu. V. Kalyuzhnyi, G. Stell, M. L. Llano-Restrepo et al., J. Chem. Phys. 101, 7939 (1994).

[21] M. S. Wertheim, J. Stat. Phys. 42, 459; 477 (1986).

[22] M. S. Wertheim, J. Chem. Phys. 85, 2929 (1986).

[23] M. S. Wertheim, J. Chem. Phys. 87, 7323 (1987).

[24] M. F. Holovko, Yu. V. Kalyuzhnui, Mol. Phys. 73, 1145 (1991).

[25] Yu. V. Kalyuzhnyi, M. F. Holovko, A. D. J. Haymet, J. Chem. Phys. 95, 9151 (1991).

[26] M. F. Holovko, I. A. Protsykevytsh, Yu. V. Kalyuzhnyi, Chem. Phys. Lett. 215, 1 (1993).

[27] G. Jackson, W. G. Chapman, K. E. Gubbins, Mol. Phys. 65, 1 (1988).

[28] C. G. Joslin, C. G. Gray, W. G. Chapman, K. E. Gubbins, Mol. Phys. 62, 843 (1987).

[29] Yu. V. Kalyuzhnyi, V. Vlachy, M. F. Holovko, G. Stell, J. Chem. Phys. 102, 5770 (1995).

[30] Yu. V. Kalyuzhnyi, G. Stell, Mol. Phys. 78, 1247 (1993).

[31] E. Kierlik, M. L. Rosinberg, J. Chem. Phys. 100, 1716 (1994).

[32] E. Kierlik, M. L. Rosinberg, J. Chem. Phys. 100, 3181 
(1994).

[33] E. Kierlik, M. L. Rosinberg, J. Chem. Phys. 102, 2141 (1995).

[34] M. F. Holovko, E. V. Vakarin, Mol. Phys. 85, 1057 (1995).

[35] M. F. Holovko, E. V. Vakarin, Yu. Ya. Duda, Chem. Phys. Lett. 233, 420 (1995).

[36] D. Henderson, F. F. Abraham, J. A. Barker, Mol. Phys. 31, 1291 (1976).

[37] D. Henderson, in: Fundamentals of Inhomogeneous Flu$i d s$ (Edited by D. Henderson), M. Dekker, NY, 1992, Ch. 4.

[38] O. Pizio, D. Henderson, S. Sokołowski, J. Phys. Chem. 99, 2408 (1995).

[39] O. Pizio, D. Henderson, S. Sokołowski, J. Coll. Interface Sci. 173, 254 (1995).

[40] D. Henderson, S. Sokołowski, O. Pizio, J. Chem. Phys. 102, 9048 (1995).

[41] O. Pizio, D. Henderson, S. Sokołowski, Mol. Phys. 85, 407 (1995).

[42] Yu. V. Kalyuzhnyi, O. Pizio, S. Sokołowski, Chem. Phys. Lett. 242, 297 (1995).

[43] S. Sokołowski, D. Henderson, A. Trokhymchuk, O. Pizio, Physica A 220, 24 (1995).

[44] O. Pizio, A. Trokhymchuk, S. Sokołowski, Mol. Phys. 86,
649 (1995).

[45] D. Henderson, S. Sokołowski, O. Pizio, Czech. Journ. Phys. 46, 67 (1996).

[46] D. Henderson, S. Sokołowski, A. Trokhymchuk, J. Chem. Phys. 103, 4693 (1995).

[47] R. Baxter, J. Chem. Phys. 52, 4559 (1970).

[48] Yu. V. Kalyuzhnyi, Yu. Ya. Duda, Cond. Matt. Phys. (Lviv) 2, 20 (1993).

[49] D. Henderson, S. Sokolowski, A. D. Trokhymchuk, Canad. J. Phys. 74, 65 (1996).

[50] A. Trokhymchuk, O. Pizio, D. Henderson, S. Sokolowski, Mol. Phys. 86, 53 (1995).

[51] A. Van Blaaderen, A. Vrij, J. Coll. and Interface Sci. 156, 1 (1993).

[52] J. Wong, private communication.

[53] D. Henderson, A. Trokhymchuk, O. Pizio, Chem. Phys. Lett. 245, 615 (1995).

[54] P. Marsh, G. Rickayzen, M. Calleja, Mol. Phys. 84, 799 (1995).

[55] W. Steele, Surf. Sci. 36, 317 (1973).

[56] S. Sokolowski, J. Coll. and Interface Sci. 88, 129 (1982).

[57] O. Pizio, S. Sokolowski, Phys. Rev. E 53, 820 (1996).

[58] O. Pizio, M. F. Holovko, Preprint Inst. Cond. Matt. Physics, No ICMP-91-2Y, Lviv, 1991.

\section{АДСОРБЩЯ ДИМЕРИЗОВАНИХ РІДИН БІЛЯ ПОВЕРХНІ ТВЕРДОГО ТІЛА. МОДЕЛЬ ПРОНИКНЕННЯ ТВЕРДИХ СФЕР}

\footnotetext{
Д. Гендерсон ${ }^{1}$, О. Пізіо ${ }^{2, *}$, С. Соколовскі ${ }^{3}$, А. Трохимчук ${ }^{2, *}$

${ }^{1}$ Факультет хімї і біологї Браям Янг, Прово, Юта 84602, США

${ }^{2}$ Iнститут хіміз УНАМ, Койоакан, 04510, Мехіко, Мексика

${ }^{3}$ Факультет хімї, Університет Марї Склодовсъкой-Кюрі, Люблін 20031, Полъща

* Постійна адреса: Інститут фізики конденсованих систем НАН Украйни, 290011 Лъвів, Украйна

Зроблено огляд недавніх досліджень неоднорідних асоціативних рідин. Розгляд обмежено випадком адсорбції димеризованих рідин біля поверхні твердого тіла. Розглядаються кристалічні або позбавлені структури поверхні, які можуть бути реактивними або нереактивними. Основними методами, що застосовуються, є методи інтегрального рівняння, проте також представлено деякі результати, отримані за допомогою методу Монте-Карло.
} 